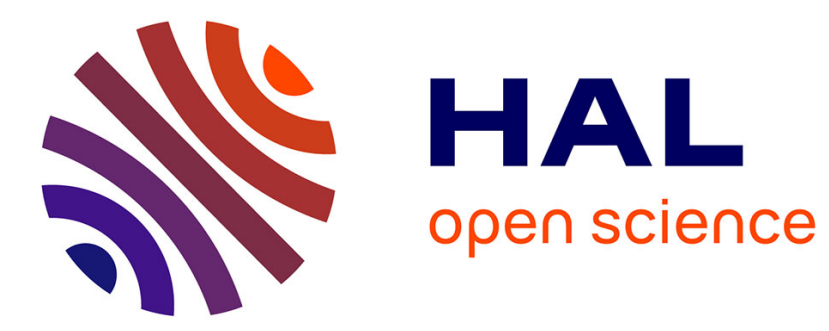

\title{
Widely linear FRESH receiver for SAIC/MAIC with frequency offsets
}

Rémi Chauvat, Pascal Chevalier, Jean-Pierre Delmas

\section{To cite this version:}

Rémi Chauvat, Pascal Chevalier, Jean-Pierre Delmas. Widely linear FRESH receiver for SAIC/MAIC with frequency offsets. ISWCS 2015: 12th International Symposium on Wireless Communication Systems, Aug 2015, Bruxelles, Belgium. 10.1109/ISWCS.2015.7454402 . hal-01254987

\section{HAL Id: hal-01254987 \\ https://hal.science/hal-01254987}

Submitted on 13 Jan 2016

HAL is a multi-disciplinary open access archive for the deposit and dissemination of scientific research documents, whether they are published or not. The documents may come from teaching and research institutions in France or abroad, or from public or private research centers.
L'archive ouverte pluridisciplinaire HAL, est destinée au dépôt et à la diffusion de documents scientifiques de niveau recherche, publiés ou non, émanant des établissements d'enseignement et de recherche français ou étrangers, des laboratoires publics ou privés. 


\title{
Widely Linear FRESH Receiver for SAIC/MAIC with Frequency Offsets
}

\author{
Rémi Chauvat*†, Pascal Chevalier*†, Jean-Pierre Delmas ${ }^{\ddagger}$ \\ ${ }^{*}$ CNAM, CEDRIC Laboratory, 292 rue Saint-Martin, 75003 Paris, France \\ ${ }^{\dagger}$ Thales-Communications-Security, HTE/AMS/TCP, 4 av. des Louvresses, 92622 Gennevilliers, France \\ ${ }^{\ddagger}$ Telecom SudParis, UMR CNRS 5157, 9 rue Charles Fourier, 91011 Evry, France
}

\begin{abstract}
Widely linear (WL) receivers are able to fulfill single antenna interference cancellation (SAIC) of one rectilinear (R) (ASK, BPSK) or quasi-rectilinear (QR) (MSK, GMSK, OQAM) co-channel interference (CCI), a function which is operational in GSM handsets in particular. However, in most cases, SAIC technology loses its efficiency if the residual frequency offset (FO) of the CCI is above a very small fraction of the baud rate. It may be the case for airborne communications, due to high differential Doppler shifts. It may also be the case if we try to use SAIC/MAIC receivers to mitigate intrinsic inter-carrier interference (ICI) of FBMC-OQAM waveforms, which are candidate for $5 \mathrm{G}$ networks, and for which the ICI FO is equal to $50 \%$ of the (real) baud rate. In this context, the purpose of this paper is twofold. The first one is to extend, for an arbitrary propagation channel and from a MLSE-based approach, the SAIC/MAIC concept to $R$ or QR signals with differential FO using WL FRESH filtering. The second one is to analyse both analytically and by simulations the impact of the residual CCI FO on the performance of the proposed SAIC/MAIC receiver.
\end{abstract}

\section{INTRODUCTION}

These two last decades, since the pioneer works on the subject [1-4], WL filtering has aroused a great interest for second-order (SO) non-circular signals [5] in many areas. Nevertheless, the subject which has attracted the greatest attention is CCI mitigation in radiocommunication networks using $\mathrm{R}$ or $\mathrm{QR}$ modulations. Let us recall that $\mathrm{R}$ modulations correspond to mono-dimensional modulations such as ASK or BPSK modulations, whereas QR modulations are complex modulations corresponding, after a simple derotation operation [6], to a complex filtering of an $\mathrm{R}$ modulation. Examples of QR modulations are MSK, GMSK or OQAM modulations. One remarkable property of WL filtering is its ability to fulfill SAIC of one R or QR multi-user (MU) CCI, allowing the separation of two users from only one receive antenna [68]. The effectiveness of this technology jointly with its low complexity are the reasons why it is operational in most of GSM handsets, generating significant network's capacity gains for the GSM system [8], [9]. Extension of the SAIC technology to a multi-antenna reception is called multiple antenna interference cancellation (MAIC). However, it has been shown recently in [10], in the GSM context, that to be efficient in practice, SAIC/MAIC MMSE-like receivers require that the residual FO of the CCI, omnipresent in practice, remains lower than a very small fraction (around 0.0001) of the baud rate. This may not be verified for airborne communications, due to high differential Doppler shifts between the signal of interest (SOI) and the CCI. This is no more verified in the context of filter bank multi-carrier (FBMC) waveforms, coupled with OQAM modulation, which are considered as promising candidates for the $5 \mathrm{G}$ mobile networks in particular [11]. Indeed, the intrinsic ICI of FBMC-OQAM waveforms, which are difficult to remove for highly frequency selective channels or for MIMO systems, have FO corresponding to multiple of $50 \%$ of the (real) baud rate. This may prevent current SAIC/MAIC receivers to remove efficiently this intrinsic ICI. Note that the scarce WL filtering based solutions available for this problem [12], [13] or for CCI mitigation [14] do not exploit the full potential of SAIC/MAIC receivers.

In this context, the purpose of this paper is twofold. The first one is to extend, in an efficient original way and for arbitrary propagation channels, the SAIC/MAIC concept to $\mathrm{R}$ or QR signals with differential FO using WL FRESH filtering. The second one is to analyse, both analytically and by simulations, the impact of the residual CCI FO on the performance of the proposed SAIC/MAIC receiver. To these aims, we adopt the continuous-time (CT) pseudo maximum likelihood sequence estimation (MLSE) approach introduced recently in [15]. The CT approach allows us to exploit the full potential of the receiver by removing both the filtering structure constraints imposed by a discrete-time (DT) approach and the possible influence of the sampling rate. Note that while [2] introduces the basis of WL FRESH filtering for estimation purposes, WL FRESH filtering for equalization/demodulation in presence of CCI has been considered in [16-19] for $R$ signals and in [20-22] for QR signals. While [18], [21] concern DS-CDMA systems, [17] considers blind processing whereas [16], [19] and [22] use DT MMSE approaches. Reference [20] considers a DT MLSE approach at the symbol rate without any FO. Besides, no analytical performance results allowing to understand the impact of residual CCI FO is presented in these papers. This prevents the good understanding of both the possibilities and limitations of WL FRESH receivers for demodulation purpose.

\section{MODELS AND STATISTICS}

\section{A. Observation model and SO statistics}

We consider an array of $N$ narrow-band antennas receiving the contribution of a SOI, which may be $\mathrm{R}$ or $\mathrm{QR}$, one MU CCI, having the same nature (R or QR) as the SOI, and a background noise. The vector of complex amplitudes of the data at the output of these antennas after frequency synchronization can then be written as

$$
\begin{aligned}
\mathbf{x}(t) & =\sum_{k}\left[a_{k} \mathbf{g}(t-k T)+f_{k}\left(v(t-k T) e^{j 2 \pi \Delta_{f} t}\right) * \mathbf{h}_{I}(t)\right]+\mathbf{u}(t) \\
& =\sum_{k} a_{k} \mathbf{g}(t-k T)+\sum_{k} f_{k} e^{j 2 \pi \Delta_{f} k T} \mathbf{g}_{I_{o}}(t-k T)+\mathbf{u}(t) \\
& \triangleq \sum_{k} a_{k} \mathbf{g}(t-k T)+\mathbf{n}(t) .
\end{aligned}
$$

Here, $\left(a_{k}, f_{k}\right)=\left(b_{k}, e_{k}\right)$ for $\mathrm{R}$ signals whereas $\left(a_{k}, f_{k}\right)=$ $\left(j^{k} b_{k}, j^{k} e_{k}\right)$ for QR signals, where $b_{k}$ and $e_{k}$ are real-valued 
zero-mean i.i.d. r.v., corresponding to the SOI and CCI symbols respectively for $\mathrm{R}$ signals and directly related to the SOI and CCI symbols respectively for QR signals [7], [23], $T$ is the symbol period for R, MSK and GMSK signals and half the symbol period for OQAM signals, $\mathbf{g}(t)=v(t) * \mathbf{h}(t)$ is the impulse response of the SOI global channel, $*$ is the convolution operation, $v(t)$ and $\mathbf{h}(t)$ are the impulse responses of the SOI pulse shaping filter and propagation channel respectively, $\Delta_{f}$ is the residual FO of the CCI, which is assumed to be known, $\mathbf{g}_{I_{o}}(t)=v_{o}(t) * \mathbf{h}_{I}(t)$ where $v_{o}(t)=v(t) e^{j 2 \pi \Delta_{f} t}$ and $\mathbf{h}_{I}(t)$ is the impulse response of the propagation channel of the $\mathrm{CCI}, \mathbf{u}(t)$ is the background noise vector, assumed zero-mean, circular, stationary, temporally and spatially white and $\mathbf{n}(t)$ is the total noise vector composed of the CCI and background noise. Note that model (1) with $\left(a_{k}, f_{k}\right)=\left(j^{k} b_{k}, j^{k} e_{k}\right)$ is exact for MSK and OQAM signals whereas it is only an approximated model for GMSK signals.

The SO statistics of $\mathbf{n}(t)$ are characterized by the two correlation matrices $\mathbf{R}_{n}(t, \tau)$ and $\mathbf{C}_{n}(t, \tau)$, defined by

$$
\begin{aligned}
\mathbf{R}_{n}(t, \tau) \triangleq \mathrm{E}\left[\mathbf{n}(t+\tau / 2) \mathbf{n}^{H}(t-\tau / 2)\right] \\
\mathbf{C}_{n}(t, \tau) \triangleq \mathrm{E}\left[\mathbf{n}(t+\tau / 2) \mathbf{n}^{T}(t-\tau / 2)\right]
\end{aligned}
$$

where $(.)^{T}$ and $(.)^{H}$ mean transpose and conjugate transpose respectively. Using (1), it is easy to verify that for both $\mathrm{R}$ and $\mathrm{QR}$ signals, $\mathbf{R}_{n}(t, \tau)$ is a periodic function of $t$ with period equal to $T$. In a same way, it is easy to show that $\mathbf{C}_{n}(t, \tau)=\mathbf{C}_{n}^{\prime}(t, \tau) e^{j 4 \pi \Delta_{f} t}$ where $\mathbf{C}_{n}^{\prime}(t, \tau)$ is a periodic function of $t$ with period equal to $T$ and $2 T$ for $\mathrm{R}$ and $\mathrm{QR}$ signals respectively. Matrices $\mathbf{R}_{n}(t, \tau)$ and $\mathbf{C}_{n}(t, \tau)$ have then Fourier series expansions given by

$$
\begin{aligned}
& \mathbf{R}_{n}(t, \tau)=\sum_{\alpha_{i}} \mathbf{R}_{n}^{\alpha_{i}}(\tau) e^{j 2 \pi \alpha_{i} t} \\
& \mathbf{C}_{n}(t, \tau)=\sum_{\beta_{i}} \mathbf{C}_{n}^{\beta_{i}}(\tau) e^{j 2 \pi \beta_{i} t} .
\end{aligned}
$$

Here, $\alpha_{i}$ and $\beta_{i}$ are the first and second SO cyclic frequencies of $\mathbf{n}(t)$ such that $\alpha_{i}=i / T(i \in \mathbb{Z})$ for both $\mathrm{R}$ and QR signals, whereas $\beta_{i}=i / T+2 \Delta_{f}$ and $\beta_{i}=(2 i+1) / 2 T+2 \Delta_{f}(i \in \mathbb{Z})$ for $\mathrm{R}$ and QR signals respectively [24], $\mathbf{R}_{n}^{\alpha_{i}}(\tau)$ and $\mathbf{C}_{n}^{\beta_{i}}(\tau)$ are the first and second cyclic correlation matrices of $\mathbf{n}(t)$ for the cyclic frequencies $\alpha_{i}$ and $\beta_{i}$ and the delay $\tau$, defined by

$$
\begin{aligned}
\mathbf{R}_{n}^{\alpha_{i}}(\tau) & \triangleq\left\langle\mathbf{R}_{n}(t, \tau) e^{-j 2 \pi \alpha_{i} t}\right\rangle_{\infty} \\
\mathbf{C}_{n}^{\beta_{i}}(\tau) & \triangleq\left\langle\mathbf{C}_{n}(t, \tau) e^{-j 2 \pi \beta_{i} t}\right\rangle_{\infty}
\end{aligned}
$$

where $\langle\cdot\rangle_{\infty}$ is the temporal mean operation in $t$ over an infinite observation duration.

\section{B. Conventional and standard extended models}

For both $\mathrm{R}$ and $\mathrm{QR}$ signals, conventional linear processing of $\mathbf{x}(t)$ only exploits the information contained in the first zero $(\alpha=0)$ SO cyclic frequency of $\mathbf{x}(t)$.

For $\mathrm{R}$ signals, standard WL processing of $\mathbf{x}(t)$ only exploits the information contained in the first and second zero $(\alpha, \beta)=(0,0)$ SO cyclic frequencies of $\mathbf{x}(t)$ through the exploitation of the temporal mean of the first correlation matrix of the extended model $\widetilde{\mathbf{x}}(t) \triangleq\left[\mathbf{x}^{T}(t), \mathbf{x}^{H}(t)\right]^{T}$. However the most energetic second SO cyclic frequency of an R CCI is $\beta=2 \Delta_{f}$, which is non-zero for $\Delta_{f} \neq 0$. For this reason, standard WL processing of $\mathbf{x}(t)$, and thus standard SAIC/MAIC receivers [6-8], give poor performance for $\Delta_{f} \neq 0$. These performance correspond to the conventional ones if no energy is present in $\beta=0$, i.e. if $\Delta_{f} \neq i / 2 T(i \in \mathbb{Z})$.

For QR signals, a derotation preprocessing of the data is required before WL filtering to make a $\mathrm{QR}$ signal looks like a $\mathrm{R}$ one [6], [15]. Then, standard WL processing of the derotated observation vector, $\mathbf{x}_{d}(t) \triangleq j^{-t / T} \mathbf{x}(t)$ only exploits the information contained in the first and second zero $\left(\alpha_{d}, \beta_{d}\right)=(0,0)$ SO cyclic frequencies of $\mathbf{x}_{d}(t)$. This is done through the exploitation of the temporal mean of the first correlation matrix of the extended derotated model $\widetilde{\mathbf{x}}_{d}(t) \triangleq\left[\mathbf{x}_{d}^{T}(t), \mathbf{x}_{d}^{H}(t)\right]^{T}$. However the two most energetic second SO cyclic frequencies of a derotated QR CCI are $\beta_{d}=2 \Delta_{f}$ and $\beta_{d}=2 \Delta_{f}-1 / T$ [15], which are non-zero if $\Delta_{f} \neq 0$ and $\Delta_{f} \neq 1 / 2 T$ respectively. In these latter cases, for QR signals, standard WL processing of $\mathbf{x}_{d}(t)$, and thus standard SAIC/MAIC receivers [6-8], give poor performance. The latter correspond to the conventional ones if no energy is present in $\beta_{d}=0$, i.e. if $\Delta_{f} \neq i / 2 T(i \in \mathbb{Z})$.

\section{Two-input FRESH models}

For both R and QR signals, efficient SAIC/MAIC receivers have to exploit the most energetic second SO cyclic frequencies of the CCI. This corresponds to the second SO cyclic frequency $\beta=2 \Delta_{f}$ of $\mathbf{x}(t)$ for $\mathrm{R} \mathrm{CCI}$ and to at least one of the two second SO cyclic frequencies $\beta_{d}=2 \Delta_{f}$ and $\beta_{d}=2 \Delta_{f}-1 / T$ of $\mathbf{x}_{d}(t)$ for QR CCI. We propose to exploit the information contained in $\beta=2 \Delta_{f}$ and in $\beta_{d}=2 \Delta_{f}$ for an $\mathrm{R}$ and a QR CCI respectively. This is done through the exploitation of the two-input FRESH observation vectors $\mathbf{x}_{F_{2}}(t)$, for R CCI, and $\mathbf{x}_{d F_{2}}(t)$, for QR CCI, defined by

$$
\begin{aligned}
\mathbf{x}_{F_{2}}(t) & \triangleq\left[\mathbf{x}^{T}(t), e^{j 4 \pi \Delta_{f} t} \mathbf{x}^{H}(t)\right]^{T} \\
& =\sum_{k} b_{k} \mathbf{g}_{F_{2}, k}(t-k T)+\mathbf{n}_{F_{2}}(t) \\
\mathbf{x}_{d F_{2}}(t) & \triangleq\left[\mathbf{x}_{d}^{T}(t), e^{j 4 \pi \Delta_{f} t} \mathbf{x}_{d}^{H}(t)\right]^{T} \\
& =\sum_{k} b_{k} \mathbf{g}_{d F_{2}, k}(t-k T)+\mathbf{n}_{d F_{2}}(t) .
\end{aligned}
$$

Here, $\quad \mathbf{n}_{F_{2}}(t) \triangleq\left[\mathbf{n}^{T}(t), e^{j 4 \pi \Delta_{f} t} \mathbf{n}^{H}(t)\right]^{T}, \quad \mathbf{n}_{d F_{2}}(t) \triangleq$ $\left[\mathbf{n}_{d}^{T}(t), e^{j 4 \pi \Delta_{f} t} \mathbf{n}_{d}^{H}(t)\right]^{T}, \quad \mathbf{n}_{d}(t) \triangleq j^{-t / T} \mathbf{n}(t)$, $\mathbf{g}_{F_{2}, k}(t) \triangleq\left[\mathbf{g}^{T}(t), e^{j 4 \pi \Delta_{f}(t+k T)} \mathbf{g}^{H}(t)\right]^{T}, \quad \mathbf{g}_{d F_{2}, k}(t) \triangleq$ $\left[\mathbf{g}_{d}^{T}(t), e^{j 4 \pi \Delta_{f}(t+k T)} \mathbf{g}_{d}^{H}(t)\right]^{T}, \mathbf{g}_{d}(t) \triangleq j^{-t / T} \mathbf{g}(t)$.

\section{GENERIC PSEUDO-MLSE RECEIVER}

To extend, in an efficient original way and for an arbitrary propagation channel, the SAIC/MAIC concept to $\mathrm{R}$ or $\mathrm{QR}$ signals with differential FO, we use the CT pseudo-MLSE approach, introduced recently in [15], and we apply it to the models (8) and (9) respectively.

\section{A. Pseudo-MLSE approach}

As the CT MLSE receiver in a cyclostationary and noncircular total noise is very challenging to derive and even probably impossible to implement, we adopt the CT pseudoMLSE approach introduced in [15]. It consists in computing the CT MLSE receiver from $\mathbf{x}_{F_{2}}(t)$ (R signals) or $\mathbf{x}_{d F_{2}}(t)(\mathrm{QR}$ signals), assuming that the associated two-input FRESH total noise $\mathbf{n}_{F_{2}}(t)$ (R signals) or $\mathbf{n}_{d F_{2}}(t)$ (QR signals), is Gaussian, circular and stationary. 


\section{B. Generic pseudo-MLSE receiver}

We denote by $\mathbf{x}_{F_{M}}(t)$ and $\mathbf{n}_{F_{M}}(t)$ (or $\mathbf{x}_{d F_{M}}(t)$ and $\left.\mathbf{n}_{d F_{M}}(t)\right)$ the generic $M(M=1,2)$ input FRESH observation and associated FRESH total noise vectors for R signals (or QR signals) respectively. We assume that $\mathbf{x}_{F_{1}}(t)$ and $\mathbf{n}_{F_{1}}(t)$ (or $\mathbf{x}_{d F_{1}}(t)$ and $\mathbf{n}_{d F_{1}}(t)$ ) correspond to $\mathbf{x}(t)$ and $\mathbf{n}(t)$ (or $\mathbf{x}_{d}(t)$ and $\left.\mathbf{n}_{d}(t)\right)$ respectively. Thus for $M=1$, we consider conventional linear receivers whereas for $M=2$ we consider twoinput WL FRESH receivers. Assuming a stationary, circular and Gaussian generic FRESH total noise $\mathbf{n}_{F_{M}}(t)$ (or $\mathbf{n}_{d F_{M}}(t)$ ), it is shown in [25], [26] that the sequence $\widehat{\mathbf{b}} \triangleq\left(\widehat{b}_{1}, \ldots, \widehat{b}_{K}\right)$ which maximizes its likelihood from $\mathbf{x}_{F_{M}}(t)$ (or $\mathbf{x}_{d F_{M}}(t)$ ) is the one which minimizes the following criterion ${ }^{1}$ :

$C(\mathbf{b})=\int\left[\mathbf{x}_{F_{M}}(f)-\mathbf{s}_{F_{M}}(f)\right]^{H}\left[\mathbf{R}_{n_{F_{M}}}^{0}(f)\right]^{-1}\left[\mathbf{x}_{F_{M}}(f)-\mathbf{s}_{F_{M}}(f)\right] d f$.

Here, $\mathbf{R}_{n_{F_{M}}}^{0}(f)$ is the Fourier transform of (6), where $\alpha_{i}$ and $\mathbf{n}(t)$ are replaced by 0 and $\mathbf{n}_{F_{M}}(t)$ respectively, whereas $\mathbf{s}_{F_{M}}(f) \triangleq \sum_{k=1}^{K} b_{k} \mathbf{g}_{F_{M}, k}(f) e^{-j 2 \pi f k T}$, where $\mathbf{g}_{F_{M}, k}(f)$ corresponds to $\mathbf{g}(f)$ for $M=1$. Considering only terms that depend on the symbols $b_{k}$, the minimization of (10) is equivalent to that of the metric:

$$
\Lambda(\mathbf{b})=\sum_{k=1}^{K} \sum_{k^{\prime}=1}^{K} b_{k} b_{k^{\prime}} r_{k, k^{\prime}}-2 \sum_{k=1}^{K} b_{k} z_{F_{M}}(k)
$$

where $z_{F_{M}}(k) \triangleq \operatorname{Re}\left[y_{F_{M}}(k)\right]$ and where the sampled output $y_{F_{M}}(k)$ and $r_{k, k^{\prime}}$ are defined by

$$
\begin{aligned}
& y_{F_{M}}(k)=\int \mathbf{g}_{F_{M}, k}^{H}(f)\left[\mathbf{R}_{n_{F_{M}}}^{0}(f)\right]^{-1} \mathbf{x}_{F_{M}}(f) e^{j 2 \pi f k T} d f \\
& r_{k, k^{\prime}}=\int \mathbf{g}_{F_{M}, k}^{H}(f)\left[\mathbf{R}_{n_{F_{M}}}^{0}(f)\right]^{-1} \mathbf{g}_{F_{M}, k^{\prime}}(f) e^{j 2 \pi f\left(k-k^{\prime}\right) T} d f .
\end{aligned}
$$

For QR signals, the results are obtained by replacing in (10), (12) and (13) $\mathbf{x}_{F_{M}}(t), \mathbf{n}_{F_{M}}(t)$ and $\mathbf{g}_{F_{M}, k}(t)$ by $\mathbf{x}_{d F_{M}}(t)$, $\mathbf{n}_{d F_{M}}(t)$ and $\mathbf{g}_{d F_{M}, k}(t)$ respectively.

\section{Interpretation of the generic pseudo-MLSE receiver}

We deduce from (12) that $y_{F_{M}}(k)$ is the sampled version, at time $t=k T$, of the output of the filter whose frequency response is

$$
\mathbf{w}_{F_{M}, k}^{H}(f) \triangleq\left(\left[\mathbf{R}_{n_{F_{M}}}^{0}(f)\right]^{-1} \mathbf{g}_{F_{M}, k}(f)\right)^{H}
$$

and whose input is $\mathbf{x}_{F_{M}}(t)$. The structure of the generic $M$ input pseudo-MLSE receiver $(M=1,2)$ is then depicted at Fig. 1. It is composed of the WL FRESH filter (14), which reduces to a time-invariant (TI) linear filter for conventional receivers, followed by a sampling at the symbol rate, a real part capture and a decision box implementing the Viterbi algorithm, since $r_{k, k^{\prime}}^{*}=r_{k^{\prime}, k}$. A similar scheme is obtained for QR signals from the derotated variables.

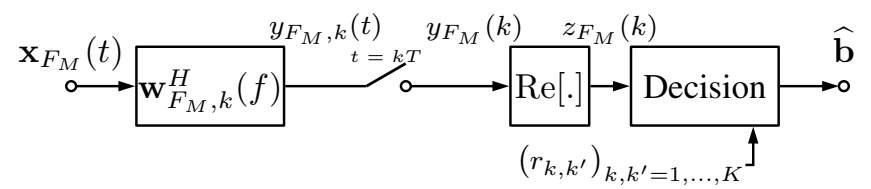

Fig. 1. Structure of the $M$ input pseudo-MLSE receiver $(M=1,2)$

\footnotetext{
${ }^{1}$ All Fourier transforms of vectors $\mathbf{x}$ and matrices $\mathbf{X}$ use the same notation
} where $t$ or $\tau$ is simply replaced by $f$.

\section{Implementation of the generic pseudo-MLSE receiver}

The implementation of the generic pseudo-MLSE receiver requires the knowledge or the estimation of $\mathbf{g}_{F_{M}, k}(f)$ and $\mathbf{R}_{n_{F_{M}}}^{0}(f)$ for each frequency $f$. This requires the knowledge of $\left(\mathbf{h}(t), \mathbf{h}_{I}(t), N_{0}, \Delta_{f}\right)$ for $M=1,2$ where $N_{0}$ is the power spectral density of the background noise.

\section{E. SINR at the output of the generic pseudo-MLSE receiver}

For real-valued symbols $b_{k}$, the symbol error rate (SER) at the output of the generic $M$ input $(M=1,2)$ pseudoMLSE receiver is directly linked to the signal to interference plus noise ratio (SINR) on the current symbol before decision, i.e. at the output $z_{F_{M}}(n)$ [27, Sec 10.1.4], while the intersymbol interference is processed by the decision box. For this reason, we compute the general expression of the output SINR hereafter and we will analyse its variations in section IV for $\mathrm{R}$ signals only, due to space limitations. As $\mathbf{n}_{F_{M}}(t)$ is cyclostationary and non-circular, the filter (14) does not maximizes the output SINR and can only be considered as a generic $M$ input pseudo-matched filter. It is easy to verify from (1), (8), (9), (12) and (13) that $z_{F_{M}}(n)$ can be written as

$$
z_{F_{M}}(n)=b_{n} r_{n, n}+\sum_{k \neq n} b_{k} \operatorname{Re}\left[r_{n, k}\right]+z_{n, F_{M}}(n)
$$

where the real-valued sample $z_{n, F_{M}}(n)$ is defined by the real part of (12) for $k=n$ with $\mathbf{n}_{F_{M}}(f)$ instead of $\mathbf{x}_{F_{M}}(f)$. Defining $\pi_{b} \triangleq \mathrm{E}\left[b_{n}^{2}\right]$, the SINR on the current symbol $n$ is then given by

$$
\operatorname{SINR}_{F_{M}, n} \triangleq \pi_{b} r_{n, n}^{2} / \mathrm{E}\left[z_{n, F_{M}}^{2}(n)\right] .
$$

\section{SINR ANALYSIS}

\section{A. Assumptions}

In this section, we analyse, for $\mathrm{R}$ signals, both analytically and by simulations, the impact of the different parameters, and of the residual CCI FO in particular, on the SINR at the output of the $M(M=1,2)$ input pseudo-MLSE receiver. For this purpose, we consider the model (1) for R signals and we assume a raised cosine pulse shaping filter $v(t)$ with a roll-off $\gamma$. The SOI and CCI have the same bandwidth, $B=(1+\gamma) / T$, and spectrally overlap if $0 \leq\left|\Delta_{f}\right| \leq B$, (i.e. if $0 \leq\left|\Delta_{f}\right| T \leq$ $1+\gamma)$ as illustrated in Fig. 2, what we assume in the following.

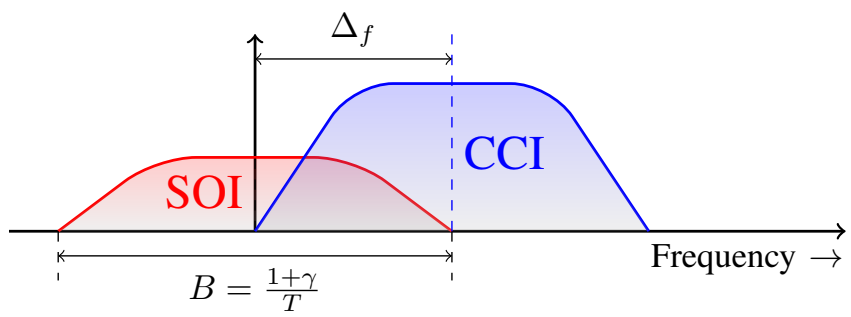

Fig. 2. Spectral representation of the SOI and CCI

Moreover, to easily describe the behavior and quantify the performance of the generic $M$ input pseudo-MLSE receiver, we limit the analysis to deterministic propagation channels with no delay spread such that

$$
\mathbf{h}(t)=\mu \delta(t) \mathbf{h} \quad \text { and } \quad \mathbf{h}_{I}(t)=\mu_{I} \delta\left(t-\tau_{I}\right) \mathbf{h}_{I} .
$$

Here, $\mu$ and $\mu_{I}$ control the amplitude of the SOI and CCI, $\delta(t)$ is the Dirac pulse, $\tau_{I}$ is the delay of the CCI with respect to 
the SOI whereas $\mathbf{h}$ and $\mathbf{h}_{I}$, such that $\mathbf{h}^{H} \mathbf{h}=\mathbf{h}_{I}^{H} \mathbf{h}_{I}=N$, are the channel vectors of the SOI and CCI.

\section{B. SINR computations and analysis for a zero roll-off}

Under the previous assumptions, analytical interpretable expressions of the $\operatorname{SINR}_{F_{M}, n}$ (16) are only possible for a zero roll-off $\gamma$ which is assumed in this subsection. Otherwise, the computation of (16) can only be done numerically, by computer simulations, and will be discussed in subsection IV. C. For a zero roll-off, the quantities $\pi_{s} \triangleq \mu^{2} \pi_{b}, \pi_{I} \triangleq \mu_{I}^{2} \pi_{e}$ and $\eta_{2}=N_{0}$ correspond to the mean power of the SOI, the CCI (for $\Delta_{f}=0$ ) and the background noise per antenna at the output of the pulse shaping matched filter respectively, where $\pi_{e} \triangleq \mathrm{E}\left[e_{n}^{2}\right]$. We then denote by $\varepsilon_{s}$ and $\varepsilon_{I}$ the quantities $\varepsilon_{s} \triangleq \pi_{s} \mathbf{h}^{H} \mathbf{h} / \eta_{2}$ and $\varepsilon_{I} \triangleq \pi_{I} \mathbf{h}_{I}^{H} \mathbf{h}_{I} / \eta_{2}$ and by $\operatorname{SINR}_{R_{M}, n}$ the SINR (16) at the output of the $M$ input pseudo-MLSE receiver at time $n T$ for $\mathrm{R}$ signals. Moreover, we denote by $\alpha_{s I}$ the spatial correlation coefficient between the SOI and the CCI, such that $\left(0 \leq\left|\alpha_{s I}\right| \leq 1\right)$ and defined by

$$
\alpha_{s I} \triangleq \frac{\mathbf{h}^{H} \mathbf{h}_{I}}{\left[\mathbf{h}^{H} \mathbf{h}\right]^{\frac{1}{2}}\left[\mathbf{h}_{I}^{H} \mathbf{h}_{I}\right]^{\frac{1}{2}}} \triangleq\left|\alpha_{s I}\right| e^{j \phi_{s I}}
$$

where $\phi_{s I}$ is the phase of $\alpha_{s I}$. Assuming a strong CCI $\left(\varepsilon_{I} \gg\right.$ $1)$, we obtain, after tedious computations not reported here:

$$
\begin{aligned}
\operatorname{SINR}_{R_{1}, n} \approx 2 \varepsilon_{s}\left[1-\left(1-\left|\Delta_{f}\right| T\right)\left|\alpha_{s I}\right|^{2}\right] ; & \left(\left|\alpha_{s I}\right|, \Delta_{f}\right) \neq(1,0) \\
\operatorname{SINR}_{R_{1}, n} & =\frac{2 \varepsilon_{s}}{1+2 \varepsilon_{I} \cos ^{2}\left(\phi_{s I}\right)} ; \quad\left(\left|\alpha_{s I}\right|, \Delta_{f}\right)=(1,0) \\
\operatorname{SINR}_{R_{2}, n} & \approx 2 \varepsilon_{s}\left[1-\left(1-\left|\Delta_{f}\right| T\right) \frac{\left|\alpha_{s I}\right|^{2}}{2}\right] ; \quad 0.5 \leq\left|\Delta_{f}\right| T \leq 1 \\
\operatorname{SINR}_{R_{2}, n} & \approx 2 \varepsilon_{s}\left[1-\left|\alpha_{s I}\right|^{2}\left\{\frac{\left|\Delta_{f}\right| T}{2}+\left(1-2\left|\Delta_{f}\right| T\right) \cos ^{2}\left(\Psi_{s I, n}\right)\right\}\right] \\
0 & \leq\left|\Delta_{f}\right| T \leq 0.5 ; \quad\left(\left|\alpha_{s I}\right|, \Delta_{f}, \Psi_{s I, n}\right) \neq(1,0, k \pi) \\
\operatorname{SINR}_{R_{2}, n} & \approx \frac{2 \varepsilon_{s}}{1+2 \varepsilon_{I}} ; \quad\left(\left|\alpha_{s I}\right|, \Delta_{f}, \Psi_{s I, n}\right)=(1,0, k \pi)
\end{aligned}
$$

where $\Psi_{s I, n}$ is defined by

$$
\Psi_{s I, n} \triangleq \phi_{s I}+2 \pi \Delta_{f}\left(n T-\tau_{I}\right) .
$$

A receiver completely cancels the CCI as $\varepsilon_{I} \rightarrow \infty$ at time $n T$ if the associated $\mathrm{SINR}_{n}$ does not converge toward zero. We deduce from (19) that the conventional receiver cancels the CCI as long as there is a spatial $\left(\left|\alpha_{s I}\right| \neq 1\right)$ or a spectral $\left(\Delta_{f} \neq 0\right)$ discrimination between the sources. In this case, it is not sensitive to the phase of the signals and the output SINR does not depend on $n$. The SINR is maximum and equal to $2 \varepsilon_{s}$, the one obtained without CCI, if the sources are spatially orthogonal $\left(\left|\alpha_{s I}\right|=0\right)$ or with no overlap $\left(\left|\Delta_{f}\right| T=1\right)$. For $N=1,\left|\alpha_{s I}\right|=1$ and the conventional receiver performs SAIC as long as $\Delta_{f} \neq 0$ but with an output SINR which strongly decreases as the overlap between the sources strongly increases. For a complete overlap $\left(\Delta_{f}=0\right)$, (20) shows that SAIC at the output of the conventional receiver is generally no longer possible, except when $\phi_{I s}=(2 k+1) \pi / 2$, where $k$ is a positive or negative integer, i.e. when the sources are in quadrature.

Moreover, we deduce from (21) that for a spectral overlap which is less than $50 \%$ and for a strong CCI, the proposed WL FRESH receiver discriminates the sources only spatially and spectrally. It is not sensitive to the phase of signals and the output SINR does not depend on $n$. For $N=1$, it performs SAIC but with better performance than the conventional receiver as shown by the comparison of (21) and (19). For a spectral overlap which is greater than $50 \%$ and for a strong CCI, (22) shows that the proposed WL FRESH receiver discriminates the sources spatially, spectrally and by phases and the output SINR depends on the differential phase of the sources and then on $n$. It completely cancels the CCI as long as there is at least one of the three discriminations between the sources. In particular, for $N=1$, the WL FRESH receiver performs SAIC as long as there is a spectral $\left(\Delta_{f} \neq 0\right)$ or a phase $\left(\Psi_{s I, n} \neq k \pi\right)$ discrimination between sources. However, as long as the spatial discrimination between the sources is not total $\left(\left|\alpha_{s I}\right|^{2} \neq 0\right)$, (22) shows that the relative weight of the phase discrimination with respect to the spectral one increases with the overlap. In other words, the phase discrimination takes over from the spectral one when the latter becomes too weak, which generates better performance than the conventional receiver for both MAIC and SAIC. In particular, for a complete overlap $\left(\Delta_{f}=0\right),(22)$ reduces to

$$
\operatorname{SINR}_{R_{2}, n} \approx 2 \varepsilon_{s}\left[1-\left|\alpha_{s I}\right|^{2} \cos ^{2}\left(\phi_{s I}\right)\right] ;\left(\left|\alpha_{s I}\right|, \phi_{s I}\right) \neq(1, k \pi)
$$

which has been obtained in [7] and which only depends on the source differential phases for $N=1$ [28].

To compare these results obtained for $\gamma=0$ and $\varepsilon_{I} \gg 1$ from a statistical perspective, we now consider the case $N=$ 1 , for which we assume that $\varepsilon_{I} \rightarrow \infty$ and $\Psi_{s I, n}$ is a r.v. uniformly distributed on $[0,2 \pi]$. In this context, we deduce easily from (19), (21) and (22) the expected value of the output SINRs respectively given, whatever the value of $\left|\Delta_{f}\right| T$, by

$$
\begin{aligned}
& \mathrm{E}_{\left[\operatorname{SINR}_{R_{1}, n}\right]} \approx 2 \varepsilon_{s}\left|\Delta_{f}\right| T \\
& \mathrm{E}\left[\operatorname{SINR}_{R_{2}, n}\right] \approx \varepsilon_{s}\left(1+\left|\Delta_{f}\right| T\right) .
\end{aligned}
$$

In the absence of overlap $\left(\left|\Delta_{f}\right| T=1\right), \mathrm{E}\left[\mathrm{SINR}_{R_{1}, n}\right]=$ $\mathrm{E}\left[\mathrm{SINR}_{R_{2}, n}\right]=2 \varepsilon_{s}$, the SINR obtained without CCI. Otherwise $\left(\left|\Delta_{f}\right| T<1\right)$, we observe that $\mathrm{E}\left[\mathrm{SINR}_{R_{1}, n}\right]<$ $\mathrm{E}\left[\mathrm{SINR}_{R_{2}, n}\right]$, which proves the better performance of the WL FRESH receiver. Besides, as the overlap increases toward $100 \%, \mathrm{E}\left[\mathrm{SINR}_{R_{1}, n}\right]$ and $\mathrm{E}\left[\mathrm{SINR}_{R_{2}, n}\right]$ decrease toward zero and $\varepsilon_{s}$ respectively. This shows relatively stable mean performance of the WL FRESH receiver whatever the overlap, contrary to that of the conventional receiver.

\section{SINR computations and analysis for arbitrary roll-off}

To complete the previous results for arbitrary values of $\gamma$, we assume that $\phi_{s I}$ and $2 \pi \Delta_{f} \tau_{I}$ are r.v uniformly distributed on $[0,2 \pi]$ and $n$ is random and such that $0 \leq n \leq\left\lfloor\frac{1}{\Delta_{f} T}\right\rfloor$. In this context, choosing $\varepsilon_{s}=10 \mathrm{~dB}$ and $\varepsilon_{I}=20 \mathrm{~dB}$, Fig. 3 and Fig. 4 show, for $\gamma=0,0.5$ respectively, for $\mathrm{R}$ signals, $N=1, M=1,2$ and $\left|\Delta_{f}\right| T=0,0.125,0.25,0.5$, $\operatorname{Pr}\left[\left(\mathrm{SINR}_{F_{M}, n} / 2 \varepsilon_{s}\right) \mathrm{dB} \geq x \mathrm{~dB}\right] \triangleq p_{R_{M}}(x)$ as a function of $x$ (dB) where $\operatorname{Pr}[$.$] means probability.$

Note, whatever $\gamma$ and as $\left|\Delta_{f}\right| T$ decreases, much better performance of the proposed WL FRESH receiver with respect to the conventional one. For a given value of $\gamma$, note the relatively stable and very good performance of the WL FRESH receiver whatever $\left|\Delta_{f}\right| T$. For example, for $\gamma=0.5$ and $x=-3 \mathrm{~dB}$, note that $p_{R_{2}}(x)=50 \%, 50 \%, 54 \%, 100 \%$ for $\left|\Delta_{f}\right| T=0,0.125,0.25,0.5$ respectively, proving the stable performance of the WL FRESH receiver for $\left|\Delta_{f}\right| T<0.5$. Note finally, for a given value of $\left|\Delta_{f}\right| T$, the decreasing performance with increasing $\gamma$ of the conventional receiver, due to the overlap increase, and the relatively stable performance of the WL FRESH receiver. 


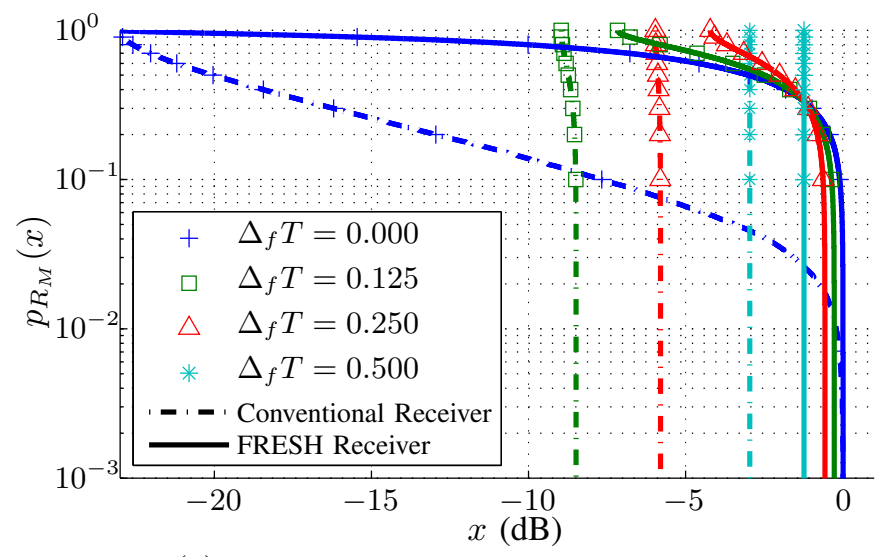

Fig. 3. $p_{R_{M}}(x)$ as a function of $x, \gamma=0, \varepsilon_{s}=10 \mathrm{~dB}, \varepsilon_{I}=20 \mathrm{~dB}$.

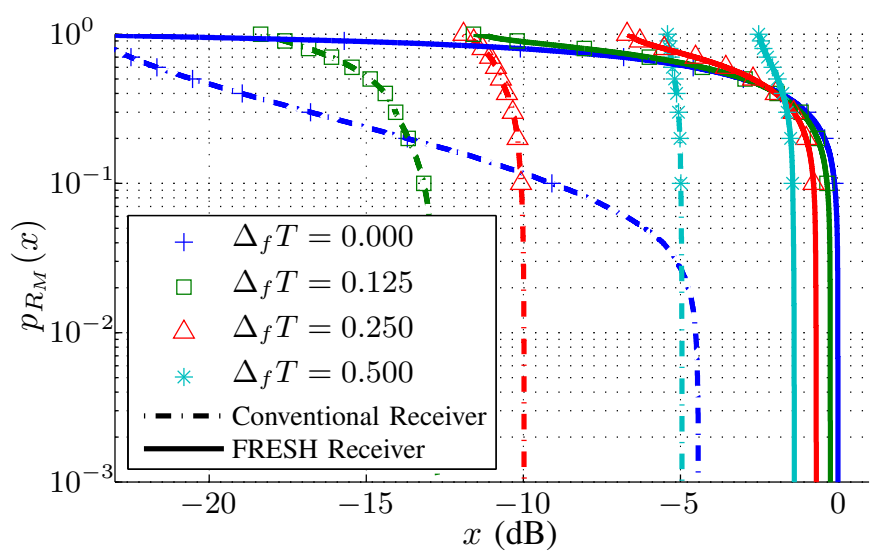

Fig. 4. $\quad p_{R_{M}}(x)$ as a function of $x, \gamma=0.5, \varepsilon_{s}=10 \mathrm{~dB}, \varepsilon_{I}=20 \mathrm{~dB}$.

\section{CONCLUSion}

The SAIC/MAIC concept has been extended, for arbitrary propagation channels and from a MLSE-based approach, to R or QR signals with differential FO using WL FRESH filtering. Performance of the proposed receiver have been analysed for $\mathrm{R}$ signals and for deterministic channels with no delay spread, both analytically and by simulations, enlightening the impact of the FO parameter. Roles of spatial, spectral and phase discrimination between the sources have been explained. Finally, it has been shown that contrary to the conventional receiver, the proposed WL FRESH receiver has good and relatively stable performance whatever the value of the FO. A detailed performance analysis for $\mathrm{QR}$ signals jointly with other approaches (MMSE, DT..) will be presented elsewhere. This may open new perspective, for intrinsic ICI mitigation of FBMC-OQAM waveforms in particular.

\section{REFERENCES}

[1] W.M. Brown and R.B. Crane, "Conjugate linear filtering", IEEE Trans. Inf. Theory, vol. 15, no. 4, pp. 462-465, July. 1969.

[2] W.A. Gardner, "Cyclic Wiener filtering: theory and method", IEEE Trans. Commun., vol. 41, no. 1, pp. 151-163, Jan. 1993.

[3] B. Picinbono and P. Chevalier, "Widely linear estimation with complex data", IEEE Trans. Signal Process., vol. 43, no. 8, pp. 2030-2033, Aug. 1995.

[4] P. Chevalier, "Optimal array processing for non stationary signals", Proc. ICASSP, pp. 2868-2871, Atlanta (USA), May 1996.

[5] B. Picinbono, "On Circularity", IEEE Trans. Signal Process., vol. 42, no. 12, pp. 3473-3482, Dec 1994.
[6] H. Trigui and D.T.M. Slock, "Performance bounds for cochannel interference cancellation within the current GSM standard", Signal Processing, Elsevier, vol. 80, pp. 1335-1346, 2000.

[7] P. Chevalier and F. Pipon, "New Insights into optimal widely linear array receivers for the demodulation of BPSK, MSK and GMSK signals corrupted by noncircular interferences - Application to SAIC", IEEE Trans. Signal Process., vol. 54, no. 3, pp. 870-883, March 2006.

[8] R. Meyer, W.H. Gerstacker, R. Schober, and J.B. Huber, "A single antenna interference cancellation algorithm for increased GSM capacity", IEEE Trans. Wireless Commun., vol. 5, no. 7, pp. 1616-1621, July 2006.

[9] A. Mostafa, R. Kobylinski, I. Kostanic, and M. Austin, "Single Antenna Interference Cancellation (SAIC) for GSM networks", IEEE Proc. Vehicular Technology Conference (VTC04), vol 2, pp. 1089-1093, Oct. 2004.

[10] JP. Delmas, S. Sallem and P. Chevalier, "Sensitivity of SAIC and MAIC Concepts to Residual Frequency Offsets", Proc. European Signal Processing Conference (EUSIPCO'10), Alborg (Denmark), Aug. 2010.

[11] G. Wunder et al, "5GNOW: Non-orthogonal asynchronous waveforms for future mobile applications", IEEE COm. Mag., pp. 97-105, Feb. 2014.

[12] M. Caus and A.I. Perez-Neira, "Multi-stream transmission for highly frequency-selective channels in MIMO-FBMC/OQAM systems", IEEE Trans. Signal Process., vol. 62, no. 4, pp. 786-796, Feb. 2014.

[13] Y. Cheng and M. Haardt, "Widely linear processing in MIMO FBMC/OQAM systems", International Symposium on Wireless Communication Systems (ISWCS'13), pp. 743-747, Aug. 2013.

[14] S. Josilo, M. Narandzic, S. Tomic, and S. Nedic, "Widely linear filtering based kindred CCI suppression in FBMC waveforms", Intern. Symposium on Wireless Communication Systems (ISWCS'14), Barcelona, Aug. 2014.

[15] P. Chevalier, R. Chauvat, and J.P. Delmas, "Quasi-rectilinear (MSK, GMSK, OQAM) co-channel interference mitigation by three inputs WL FRESH filtering", Proc. ICASSP, Brisbane (Australia), Apr. 2015.

[16] W.A. Gardner and W.A. Brown, "Frequency-Shift Filtering Theory for Adaptive Co-Channel Interference Removal", 23th Asilomar Conference on Signals, Systems and Computers, vol. 2, pp. 562-567, Nov. 1989.

[17] H.E. Wong and J.A. Chambers, "Two-stage interference immune blind equalizer which exploits cyclostationary statistics", Electronic Letters, vol. 32, no. 19, pp. 1763-1764, Sept. 1996.

[18] G. Gelli, L. Paura and A.M. Tulino, "Cyclostationarity-based filtering for narrow-band interference suppression in direct sequence spreadspectrum systems", IEEE Journal on Selected Areas in Communications, vol. 6, no. 9, pp. 1747-1755, Dec. 1998.

[19] G. Latouche, D. Pirez, P. Vila, "MMSE cyclic equalization", Proc. MILCOM, pp. 150-154, Boston, Oct. 1998.

[20] W.A. Gardner and C.W. Reed, "Making the most out of spectral redundancy in GSM: Cheap CCI suppression", Proc. ASILOMAR Conf., pp. 883-889, Pacific-Grove, 2001.

[21] A. Mirbagheri, K.N. Plataniotis, and S. Pasupathy, "An enhanced Widely Linear CDMA receiver with OQPSK modulation", IEEE Trans. Commun., vol. 54, no. 2, pp. 261-272, Feb. 2006.

[22] A.U.H. Sheikh and F. Hendessi, "FRESH-DFE: a new structure for interference cancellation", Wireless Personal Communications Journal, vol. 44, pp. 101-118, 2008.

[23] W.H. Gerstacker, R. Schober, and A. Lampe, "Receivers with widely linear processing for frequency-selective channels", IEEE Trans. Commun., vol. 51, no. 9, pp. 1512-1523, Sept. 2003.

[24] D. Vucic and M. Obradovic, "Spectral Correlation evaluation of MSK and Offset QPSK modulation", Signal Proc., vol. 78, pp. 363-367, 1999.

[25] S. Sallem, J.P. Delmas, and P. Chevalier, "Optimal SIMO MLSE receivers for the detection of linear modulation corrupted by noncircular interference," IEEE workshop on Statistical Signal Processing (SSP'2012), Ann Arbor, USA, Aug. 2012.

[26] G. Ungerboeck, "Adaptive maximum likelihood receiver for carriermodulated data transmission systems", IEEE Trans. Commun., vol. 22, no. 5, pp. 624-636, May 1974.

[27] J.G. Proakis, Digital Communications, McGraw Hill Series in Electrical and Computer Engineering, 4th Edition, 2001.

[28] R. Chauvat, P. Chevalier and J.P. Delmas, "How to make quasirectilinear signals (MSK, GMSK, OQAM) almost equivalent to rectilinear ones (BPSK, ASK) for WL filtering in the presence of CCI", ITG Workshop on Smart Antennas (WSA'15), Ilmenau, Germany, March 2015. 